\title{
Individual prediction of psychotherapy outcome in posttraumatic stress disorder using neuroimaging data
}

\author{
Paul Zhutovsky $\mathbb{D}^{1,2}$, Rajat M. Thomas ${ }^{1,2}$, Miranda Olff',3, Sanne J. H. van Rooij $\mathbb{C}^{4}$, Mitzy Kennis ${ }^{5}$,
} Guido A. van Wingen ${ }^{1,2}$ and Elbert Geuze (i) 6 ,

\begin{abstract}
Trauma-focused psychotherapy is the first-line treatment for posttraumatic stress disorder (PTSD) but 30-50\% of patients do not benefit sufficiently. We investigated whether structural and resting-state functional magnetic resonance imaging (MRI/rs-fMRI) data could distinguish between treatment responders and non-responders on the group and individual level. Forty-four male veterans with PTSD underwent baseline scanning followed by traumafocused psychotherapy. Voxel-wise gray matter volumes were extracted from the structural MRI data and resting-state networks (RSNs) were calculated from rs-fMRI data using independent component analysis. Data were used to detect differences between responders and non-responders on the group level using permutation testing, and the singlesubject level using Gaussian process classification with cross-validation. A RSN centered on the bilateral superior frontal gyrus differed between responders and non-responder groups $\left(P_{\mathrm{FWE}}<0.05\right)$ while a RSN centered on the presupplementary motor area distinguished between responders and non-responders on an individual-level with $81.4 \%$ accuracy $(P<0.001,84.8 \%$ sensitivity, $78 \%$ specificity and AUC of 0.93$)$. No significant single-subject classification or group differences were observed for gray matter volume. This proof-of-concept study demonstrates the feasibility of using rs-fMRI to develop neuroimaging biomarkers for treatment response, which could enable personalized treatment of patients with PTSD.
\end{abstract}

\section{Introduction}

Posttraumatic stress disorder (PTSD) is a psychiatric disorder that can develop after experiencing a traumatic event. It is characterized by states of re-experiencing of the traumatic event, avoidance of trauma-reminders, emotional numbing, and hyperarousal ${ }^{1}$. PTSD lifetime prevalence rates in the general population are estimated to be below $10 \%$ (varying between 1.3 to $8.8 \%$ depending on the country $)^{2,3}$ but can vary heavily in veterans (between 1.4 to $31 \%)^{4,5}$. Treatment of PTSD typically

\footnotetext{
Correspondence: Paul Zhutovsky (p.zhutovsky@amsterdamumc.nl) ${ }^{1}$ Department of Psychiatry, Amsterdam UMC, Location AMC, University of Amsterdam, Amsterdam Neuroscience, Amsterdam, The Netherlands ${ }^{2}$ Amsterdam Brain and Cognition, University of Amsterdam, Amsterdam, The Netherlands

Full list of author information is available at the end of the article.

These authors contributed equally: Guido A. van Wingen, Elbert Geuze
}

involves trauma-focused psychotherapy with or without the administration of medication such as selective serotonin reuptake inhibitors (SSRIs). Trauma-focused therapies, such as trauma-focused cognitive behavior therapy (TF-CBT) or eye movement desensitization and reprocessing (EMDR) have been suggested as first-line treatments for treating $\mathrm{PTSD}^{6,7}$. However, $30-50 \%$ of patients do not benefit sufficiently ${ }^{8}$. To improve treatment response rates it is important to better understand differences between responders and non-responders, and identify reliable predictors for treatment outcome.

PTSD is characterized as a brain disorder showing alterations in activity and connectivity of cortical and subcortical brain regions. The neurocircuitry model of PTSD suggests that PTSD pathology is characterized by hyperactivity and increased connectivity of the amygdala, the

\section{(c) The Author(s) 2019}

(c) Open Access This article is licensed under a Creative Commons Attribution 4.0 International License, which permits use, sharing, adaptation, distribution and reproduction in any medium or format, as long as you give appropriate credit to the original author(s) and the source, provide a link to the Creative Commons license, and indicate if changes were made. The images or other third party material in this article are included in the article's Creative Commons license, unless indicated otherwise in a credit line to the material. If material is not included in the article's Creative Commons license and your intended use is not permitted by statutory regulation or exceeds the permitted use, you will need to obtain permission directly from the copyright holder. To view a copy of this license, visit http://creativecommons.org/licenses/by/4.0/. 
anterior insula and the anterior cingulate cortex, decreased activity of the ventromedial prefrontal cortex (vmPFC) and hypoconnectivity between vmPFC, hippocampus and amygdala ${ }^{9-12}$. Functional connectivity of these regions can be recorded using neuroimaging techniques such as resting-state functional magnetic resonance imaging (rs-fMRI). Therefore, it is important to investigate if those alterations in rs-fMRI connectivity could be used to predict treatment-outcome and reveal biomarkers to increase the treatment-response rate. Indeed, pre-treatment group differences in fMRI activity and connectivity were observed between responders and non-responders in PTSD in several studies ${ }^{13-17}$. However, these group-level univariate analyses focus on average differences between responders and non-responders. This does not allow inference at the individual patient level, which can be achieved using multivariate supervised machine learning analyses ${ }^{18,19}$. Most importantly, performance is evaluated on new data to estimate the generalizability of the trained models, and thereby enabling the prediction of treatment outcome for new patients. Machine-learning analyses have been performed in the context of PTSD using different modalities of MRI data to distinguish between patients and controls ${ }^{20-23}$. However, only two studies to date have used machine learning analyses to predict future outcome at an individual level. One study aimed to predict clinical status two years after treatment with 12 weeks of paroxetine in a sample of 20 civilian PTSD patients ${ }^{24}$. This study used pre- and posttreatment rs-fMRI derived measures, namely amplitude of low-frequency fluctuations and whole-brain degree centrality maps, and the results showed that pre- but not post-treatment measures were able to predict remission status after two years with an accuracy of $72.5 \%$. But as all but one patient had been in remission shortly after treatment, these results reflect relapse rather than treatment outcome. In addition, one recent study used a combination of resting-state connectivity within the ventral attention network and delayed recall performance in a verbal memory task to predict the response to prolonged exposure therapy in $\sim 19$ civilian patients with $\mathrm{PTSD}^{25}$. Although the proportion of treatment nonresponders was low, the classifier still managed to distinguish the groups with $\geq 80 \%$ sensitivity and specificity.

To determine whether neuroimaging data could also predict treatment outcome in a larger sample of combat veterans with PTSD, we analyzed pre-treatment structural MRI and rs-fMRI data of 44 patients who received treatment-as-usual. This consisted of trauma-focused psychotherapy such as TF-CBT and EMDR, and clinical outcome was determined 6-8 month following the baseline fMRI scan. We previously reported pre-treatment group differences in structural ${ }^{26}$, white-matter ${ }^{27}$ and taskbased (f)MRI ${ }^{13,28}$ between responders and non- responders, as well as rs-fMRI differences between patients and controls ${ }^{29}$. In another study, we explored time-by-group interactions between remitted and persistent PTSD patients based on network measures derived from rs-fMRI ${ }^{30}$. In the current study, we focus on pretreatment MRI measures exclusively within a machinelearning paradigm. We extracted functional connectivity (FC) within resting-state networks (RSNs) using independent component analysis (ICA). ICA was chosen as the method for analyzing rs-fMRI because it provides a multivariate and data-driven way to identify multiple RSN's present in the data. It does not require the definition of seed regions and is more robust to noise ${ }^{31}$. To ensure independence between RSN identification and estimation of RSN expression for each individual patient, the ICA was performed on rs-fMRI data of sex and age matched combat controls $(n=28)$. In addition, we derived maps of regional gray matter volume using voxelbased morphometry (VBM). Abnormalities in structural MRI data have been repeatedly linked to treatmentresponse in PTSD ${ }^{26,32-35}$ and we therefore used it as a standardized and easy to acquire imaging baseline. For both the rs-fMRI and VBM data, we performed univariate inference on the group level as well as multivariate prediction on the single-subject level using Gaussian process classification (GPC) with $10 \times 10$ cross-validation. The current study is exploratory and investigated the general feasibility of structural and rs-fMRI data to predict treatment-outcome in PTSD on the single-subject level. Because of its exploratory nature, there were no a priori hypotheses formulated.

\section{Materials and methods \\ Participants}

In total 57 veterans with PTSD and 29 combat controls (CC) were included in the study. Patients were recruited from one of four outpatient clinics of the Military Mental Healthcare Organization in Utrecht, The Netherlands. PTSD diagnosis was established by a licensed psychologist or psychiatrist. The Clinician Administered PTSD scale $(\mathrm{CAPS})^{36}$ for DSM-IV ${ }^{1}$ was administered by trained research staff to quantify the total symptom severity and had to be $\geq 45$. Combat controls had to have no current psychiatric disorders and a total CAPS score $<15$. Further inclusion criteria for all subjects were deployment to a war zone and 18-60 years of age. Comorbid disorders were examined using the structured clinical interview for DSMIV (SCID-I $)^{37}$. Subjects with a history of neurological disorders, current substance dependence and contraindications for MRI scanning were excluded. From the initial 57 PTSD patients, seven were lost to follow-up, three were excluded based on excessive motion during scanning (see Supplementary information), one due to an artifact in the MRI scan, and one due to refusal of 
scanning. One additional participant was excluded as she was the only female in the sample. This lead to the final sample of 44 PTSD patients. From the CC only one subject had to be excluded based on excessive motion $(n=28)$.

After a period of six to eight months in which patients underwent treatment-as-usual consisting of traumafocused therapy (e.g., TF-CBT, EMDR) a second CAPS assessment was performed. Treatment response was defined as a $\geq 30 \%$ decrease of total CAPS score at followup with respect to the baseline assessment ${ }^{38,39}$. According to this criterion 24 PTSD veterans were defined as responder and 20 as non-responder. All participants gave written informed consent. The study was approved by the University Medical Center Utrecht ethics committee, in accordance with the declaration of Helsinki ${ }^{40}$.

\section{Clinical data analysis}

To estimate whether the $\mathrm{CC}$, responders and nonresponders differed across any demographic or clinical variables at baseline or follow-up ANOVA, Kruskal-Wallis, $\chi^{2}$, or $t$-tests were applied as appropriate. All tests were performed using the R software (version 3.5.1).

\section{Data acquisition}

All scans were obtained on a 3T MRI scanner (Philips Medical System, Best, the Netherlands). The T1-weighted high resolution MRI scan was acquired before the rs-fMRI scan with the following parameters: repetition time $(\mathrm{TR})=$ $10 \mathrm{~ms}$, echo time $(\mathrm{TE})=4.6 \mathrm{~ms}$, flip angle $=8^{\circ}, 200$ sagittal slices, field of view $(\mathrm{FOV})=240 \times 240 \times 160$, matrix size $=304 \times 299$ and voxel size $=0.8 \times 0.8 \times 0.8 \mathrm{~mm}$. The rsfMRI scan consisted of 320 T2*-weighted echo planar interleaved slices with $\mathrm{TR}=1600 \mathrm{~ms}, \mathrm{TE}=23 \mathrm{~ms}$, flip angle $=72.5^{\circ}, \mathrm{FOV}=256 \times 208 \times 120,30$ transverse slices, matrix size $=64 \times 51$, total scan time $8 \mathrm{~min}$ and $44.8 \mathrm{~s}$, $0.4 \mathrm{~mm}$ gap, acquired voxel size $=4 \times 4 \times 3.60 \mathrm{~mm}$ ). Participants were asked to focus on a fixation cross, while letting their mind wander and relax.

\section{MRI data preprocessing}

To estimate whether structural images carry information to distinguish between responders or non-responders a VBM analysis was performed. Gray matter (GM) voxelwise volume maps were computed using the SPM12 toolbox (v7219, https://www.fil.ion.ucl.ac.uk/spm/software/ spm12/).

Resting-state fMRI images were preprocessed using the advanced normalization tools (ANTs, 2.1.0, http://stnava. github.io/ANTs/) and FMRIB Software Library (FSL, 5.0.10, https://fsl.fmrib.ox.ac.uk/fsl/fslwiki/. To control for the influence of motion on the rs-fMRI data ICAAROMA was applied ${ }^{41}$. Details on the preprocessing pipelines can be found in the Supplementary information.

\section{Resting-state network identification}

Preprocessed rs-fMRI data were analyzed to determine group-level resting-state networks (RSNs). Group components with a fixed number of 70 components were estimated using a meta-ICA approach utilizing FSL's MELODIC software ${ }^{42}$ applied to the rs-fMRI data of the CC. The identification of RSNs only on the data of the CC was done to reduce the potential of overfitting during the machine learning analysis since the training and test data sets would have to be taken together when defining the RSNs if data of the PTSD patients would be included. However, a potential drawback is that the identified RSNs might not optimally represent the rs-fMRI of the PTSD patients. The number of components was fixed to 70 because it was shown to provide good insight into clinical differences of patient groups ${ }^{43}$. However, it should be noted that such a higher-order ICA can split up canonical networks into multiple sub-networks which might reduce the information present in each sub-network. The metaICA approach allows for the identification of reproducible and reliable group components ${ }^{44}$. After meta-ICA, 48 RSN's were identified using a semi-automatic approach. Thereafter, FSL's dual regression approach was used to estimate single-subject spatial representations of the corresponding group networks for all patients. Details on the implementation and rationale of the procedure can be found in the Supplementary information, and signal and noise components are illustrated in Figs. S1 and S2.

\section{Univariate analysis}

The preprocessed GM volume maps from the VBM analysis and the identified RSNs were used to investigate group differences between responders and nonresponders. Age and total intracranial volume were entered as covariates for the VBM data, while only age was used as covariate for the RSN data. The significance level was set to $P<0.05$ family-wise error (FWE) corrected and estimated using the threshold-free-clusterenhancement statistic (TFCE) ${ }^{45}$ with permutation testing (10,000 permutations) using the TFCE toolbox (r167, http://dbm.neuro.uni-jena.de/tfce/) for the VBM data. For the resting-state data, the PALM toolbox (a112, https:// fsl.fmrib.ox.ac.uk/fsl/fslwiki/PALM) was used since it allowed for permutation-based FWE correction across the whole-brain and all 48 RSNs at the same time. Both analyses accounted for two-tailed tests.

\section{Multivariate analysis}

For the multivariate single-subject classification of responders and non-responders, we used the GM volumemaps from the VBM analysis and each RSN separately. The classification was performed using a Gaussian process classifier $(\mathrm{GPC})^{46}$. Briefly, GPCs are multivariate Bayesian classifiers which allow to obtain valid 
probabilistic predictions by estimating the posterior distribution, given a pre-defined prior distribution. GPCs are a standard classifier used in machine learning for neuroimaging and has been shown to perform comparable to support vector machines ${ }^{47}$. We utilized the ability of the GPC's to provide valid probabilistic predictions to investigate posthoc the performance of the classifier when a 'reject' option is implemented (see below). Univariate feature selection was performed on the training set to reduce the initial data dimension using nested 5-fold cross-validation (see Supplementary information). The performance was estimated by calculating sensitivity, specificity, balanced accuracy, area under the receiveroperator curve (AUC) and positive/negative predictive value (PPV/NPV) using ten times repeated 10 -fold crossvalidation to avoid overfitting bias. To estimate whether our classifier performed better than chance, label permutation tests with 1000 iterations were performed. The final $P$-values were Bonferroni corrected for 49 tests.

We also investigated the performance of the GPC when an uncertainty option was allowed: utilizing the probabilistic output of the classifier, we established regions of uncertainty for which the classifier would not make a prediction. For example, with an uncertainty region of $10 \%$ any probabilistic GPC output for a new patient which lies within $45-55 \%$, would not be assigned a classification label (because the classification into responders and nonresponders would be uncertain). Only patients with a higher (or lower) probability would be assigned to a class and considered for calculation of balanced accuracy. This allowed us to investigate how well our GPC would perform if classification has only to be made if a specific level of certainty is reached and how many patients would need to be excluded to reach that level.

The code used in the analysis of the data can be made available upon request.

\section{Results}

\section{Clinical data}

Demographic information, clinical variables and outcomes of statistical tests can be found in Table 1. There was no difference in demographics between the CC, responders or non-responders, nor any clinical difference between responders and non-responders at baseline. At follow-up non-responders showed a higher total CAPS score $(\mathrm{t}(42)=7.89, P<0.001)$ and higher use of serotonin reuptake inhibitors $\left(X^{2}(1)=5.77, P=0.02\right)$.

\section{Univariate analysis}

After correction for multiple comparisons across all RSNs, the rs-fMRI analysis showed one network with significantly increased connectivity in non-responders as compared to responders (Fig. 1). The network was centered on the bilateral lateral frontal polar area and the difference was observed in the right superior frontal gyrus $\left(P_{\mathrm{FWE}}=0.04\right)$. In Fig. S4, we show all univariate groupdifferences when no FWE-correction across networks was applied, performed for illustrative purposes only. No significant group differences in GM were observed.

\section{Multivariate analysis}

GPC's trained on a network centered around the presupplementary motor area (pre-SMA) could classify nonresponders and responders with an average cross-validated balanced accuracy of $81.4 \%$ (SD: 17.2, $P_{\text {Bonferroni }}<0.05$ ) (Fig. 2a). The network showed excellent AUC (0.929, SD: $0.149)$ with high sensitivity (84.8\%, SD: 25.1$)$, moderately high specificity (78\% SD: 28.6), and high PPV/NPV (0.840/ 0.835 , SD: $0.214 / 0.262)$. No other network showed significant classification performance after Bonferroni correction was applied, including the network that showed a significant difference on the group-level in the univariate analysis. However, if no Bonferroni correction is applied this network becomes significant, as well as three additional networks. Uncorrected networks and consistently selected features are shown for illustrative purposes in Fig. S5.

To investigate which regions of the pre-SMA network were most important for the classification process we examined consistently selected voxels during the feature selection process. We tracked the selection frequency of voxels across cross-validation runs, looking at voxels which were selected in $>50 \%$ of the runs (Table 2 and Fig. 3). Regions in both hemispheres located outside the group-network were contributing to the classification performance. The largest clusters were located in the left inferior temporal gyrus $\left(\mathrm{n}_{\text {voxel }}=14\right)$, left superior frontal gyrus $\left(\mathrm{n}_{\text {voxel }}=10\right)$, and right precentral gyrus $\left(\mathrm{n}_{\text {voxel }}=9\right)$. For illustrative purposes we also computed mean correlations for responder and non-responder groups separately between average time-courses of the largest clusters $\left(\mathrm{n}_{\text {voxel }}>5\right.$, Table 2) and the subject-specific time-courses of the pre-SMA network identified by dual regression (Fig. S3). Patterns of positive, negative and no significant connectivity with the network can be observed. Note that null-connectivity voxels might still contribute to the classification by removing common noise sources from the overall pattern ${ }^{48}$.

Additionally, we provided a posthoc evaluation of what would happen if prediction would only be made for patients for which a high degree of certainty of the classifier is established. As illustrated in Fig. 2b, this ability to 'reject' patients from the classification with increasing classification certainty leads to increasing accuracy while at the same time reducing the number of patients for which the GPC can make a classification. For example, once 12 patients (27\%) with low prediction certainty of $0.41-0.59$-where 0.5 is equal probability of predictionwould be excluded, accuracy would increase to over $90 \%$. 
Table 1 Demographics and clinical data.

\begin{tabular}{|c|c|c|c|c|}
\hline & Combat Controls $(n=28)$ & Responders $(n=24)$ & Non-Responders $(n=20)$ & Test-value(df), $P$-value \\
\hline Age (mean, SD [years]) & $37.00(10.13)$ & $33.25(7.76)$ & $38.65(9.34)$ & $F(2,69)=2.057, P=0.136^{\mathrm{a}}$ \\
\hline Gender $(m / f)$ & $28 / 0$ & $24 / 0$ & $20 / 0$ & \\
\hline Handedness (left/ambidexter/right) & $2 / 3 / 23$ & $2 / 2 / 20$ & $2 / 2 / 16$ & $X^{2}(4)=0.207, P=0.995^{b}$ \\
\hline \multicolumn{5}{|l|}{ Education (median, IQR [ISCED]) } \\
\hline Own & $6[4.75,7]$ & $6[5.75,6]$ & $5.5[3,6]$ & $X^{2}(2)=4.005, P=0.135^{c}$ \\
\hline Mother & $3.5[2,6]$ & $3[2,4]$ & $3[2,6]$ & $X^{2}(2)=1.325, P=0.516^{c}$ \\
\hline Father & $5[2,6.5]$ & $3.5[2.25,7]$ & $5[2,7]$ & $x^{2}(2)=0.044, P=0.978^{c}$ \\
\hline $\begin{array}{l}\text { Time since last deployment (mean, } \\
\text { SD [years]) }\end{array}$ & $5.89(6.56)$ & $6.71(7.83)$ & $8.05(9.51)$ & $X^{2}(2)=0.218, P=0.897^{c}$ \\
\hline Number of times deployed $(1 / 2 / 3 />3)$ & $(10 / 8 / 4 / 6)$ & $(9 / 5 / 3 / 7)$ & $(8 / 3 / 6 / 2)$ & $x^{2}(2)=0.416, P=0.812$ \\
\hline FD (mean, SD) & $0.10(0.04)$ & $0.09(0.05)$ & $0.12(0.07)$ & $x^{2}(2)=3.278, P=0.194^{c}$ \\
\hline TIV (mean, SD) & & $1550.02(121.15)$ & $1528.06(166.44)$ & $\mathrm{t}(42)=-0.506, P=0.616^{\mathrm{d}}$ \\
\hline \multicolumn{5}{|l|}{ Clinical scores at baseline } \\
\hline CAPS (mean, SD) & & $71.92(15.06)$ & $69.85(11.45)$ & $\mathrm{t}(42)=0.504, P=0.617^{\mathrm{d}}$ \\
\hline \multicolumn{5}{|l|}{ Pre-treatment comorbid disorder baseline (SCID) } \\
\hline Mood disorder & & 13 & 10 & $x^{2}(1)=0.076, P=0.783^{b}$ \\
\hline Anxiety disorder & & 5 & 9 & $X^{2}(1)=2.937, P=0.087^{b}$ \\
\hline Somatoform disorder & & 1 & 1 & $X^{2}(1)=0.017, P=0.895^{b}$ \\
\hline \multicolumn{5}{|l|}{ Pre-treatment medication } \\
\hline SRI & & 5 & 7 & $X^{2}(1)=1.104, P=0.293^{b}$ \\
\hline Benzodiazepines & & 7 & 3 & $X^{2}(1)=1.247, P=0.264^{b}$ \\
\hline Antipsychotics & & 2 & 0 & $x^{2}(1)=1.746, P=0.186^{b}$ \\
\hline $\begin{array}{l}\text { Total number of treatment sessions } \\
\text { (mean, SD) }\end{array}$ & & $9.86(6.29)$ & $10.05(4.22)$ & $\mathrm{t}(38)=-0.114, P=0.910^{\mathrm{d}}$ \\
\hline \multicolumn{5}{|l|}{ Received therapy } \\
\hline TF-CBT (yes/no) & & $6 / 18$ & $10 / 10$ & $X^{2}(1)=2.946, P=0.086^{b}$ \\
\hline EMDR (yes/no) & & $20 / 4$ & $16 / 4$ & $X^{2}(1)=0.081, P=0.775^{b}$ \\
\hline \multicolumn{5}{|l|}{ Clinical scores at post-treatment } \\
\hline CAPS (mean, SD) & & $29.75(16.53)$ & $68.55(15.89)$ & $\mathrm{t}(42)=7.889, P<0.001^{\mathrm{d} *}$ \\
\hline \multicolumn{5}{|c|}{ Post-treatment comorbid disorder post-treatment (SCID) } \\
\hline Mood disorder & & 3 & 3 & $X^{2}(1)=0.096, P=0.757^{b}$ \\
\hline Anxiety disorder & & 2 & 5 & $X^{2}(1)=2.516, P=0.113^{b}$ \\
\hline Somatoform disorder & & 0 & 1 & $x^{2}(1)=1.293, P=0.256^{b}$ \\
\hline Alcohol dependency & & 0 & 2 & $x^{2}(1)=2.650, P=0.104^{b}$ \\
\hline \multicolumn{5}{|l|}{ Post-treatment medication } \\
\hline SRI & & 5 & 11 & $x^{2}(1)=5.768, P=0.016^{b_{*}}$ \\
\hline Benzodiazepines & & 5 & 1 & $x^{2}(1)=2.307, P=0.129^{b}$ \\
\hline Antipsychotics & & 2 & 2 & $x^{2}(1)=0.040, P=0.841^{b}$ \\
\hline
\end{tabular}

$S D$ standard deviation, IQR interquartile range, ISCED international scale for education, $F D$ framewise displacement, TIV total intracranial volume, CAPS clinician administered PTSD scale, SCID structured clinical interview for DSM IV Axis II disorders, SRI serotonin reuptake inhibitor, TF-CBT trauma-focused cognitive behavioral therapy, EMDR eye movement desensitization and reprocessing

${ }^{a}$ ANOVA

${ }^{b} x^{2}$

'Kruskal-Wallis

${ }^{\mathrm{d}}$ Two-sample $t$-test

${ }^{*} P<0.05$

\section{Discussion}

The present study investigated the possibility of using pre-treatment structural MRI and rs-fMRI data to predict the response to trauma-focused psychotherapy in male combat veterans with PTSD. The results showed that rsfMRI data successfully distinguished between responders and non-responders in univariate and multivariate analyses. The univariate analysis detected group differences in a network centered on the frontal pole, and the multivariate analysis predicted treatment response on an individual level using pre-SMA connectivity with an accuracy of $81.4 \%$. Whereas previous studies have focused on MRI-based treatment outcome predictors at the group level, our results suggest that single-subject prediction is also feasible. This result provides a proof-of-concept for the feasibility of developing predictive biomarkers, which could enable personalized treatment for patients with PTSD. 


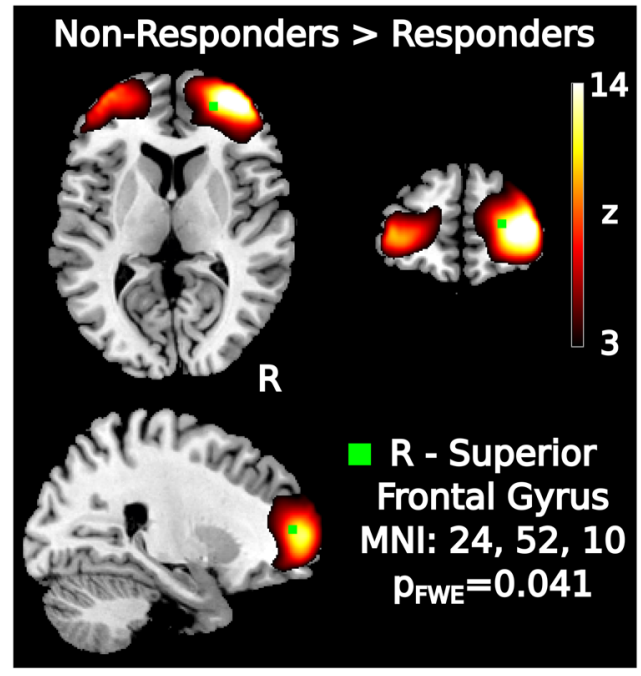

Fig. 1 Results of the group-level univariate RSN analysis. Higher resting-state connectivity was observed in non-responders than responders in the frontopolar network. Two-tailed $P$-value was corrected for whole-brain comparisons and 48 networks.

Our multivariate analysis revealed the predictive importance of the pre-SMA. This brain area is closely linked to the SMA, and is involved in motor preparation, response inhibition, and imagination ${ }^{49-51}$. Intriguingly, resting-state connectivity within this network is also predictive for the response to electroconvulsive therapy in depression $^{52}$. The main difference in results is that the network in the current study is more confined to the preSMA due to the use of ICA with 70 components instead of 32 components, which was associated with a larger network that consisted of a large part of the dorsomedial prefrontal cortex. Together, this suggests that pre-SMA connectivity may determine responsiveness to treatment, regardless of intervention and disorder.

The discovered network is different from the ventral attention network (VAN, consisting of the insula, dorsal anterior cingulate, anterior middle frontal gyrus, and supramarginal gyrus) that was recently reported. The VAN in combination with delayed recall performance in a verbal memory task could predict prolonged exposure therapy outcome in a sample of $\sim 19$ civilians with PTSD with sensitivity and specificity $\geq 80 \%{ }^{25}$. But even though both studies used rs-fMRI, the underlying biomarkers cannot readily be compared. First, the variables tested in ${ }^{25}$ were discovered by performing comparisons between healthy controls and PTSD patients, whereas we discovered the pre-SMA network from comparisons between responders and non-responders directly. Second, the authors did not investigate any other networks beyond the VAN for treatment outcome prediction. And third, the brain regions that are part of the VAN were part of distinct RSNs in our ICA analysis, whereas the VAN was considered one network in the previous study. Therefore, it remains to be tested whether VAN or pre-SMA connectivity is also predictive in other samples. Regardless, both studies demonstrate that rs-fMRI contains information that is informative for predicting psychotherapy outcome on an individual level.

The univariate group analysis showed increased connectivity in non-responders in the frontal pole. The frontal pole region (BA 10) has been implicated in a multitude of cognitive tasks, including attention, perception, language, and memory tasks ${ }^{53,54}$. Specifically, the lateral parts of the frontal pole are more associated with working memory and episodic memory retrieval while medial parts of the frontal pole were mostly involved in mentalizing, which is the reflection of your own emotions and mental states ${ }^{53,54}$. This division of the frontal pole was recently confirmed by a cytoarchitectonic parcellation indicating two distinct areas: a more lateral frontal pole area 1 (FP 1) and a more medial frontal pole area 2 (FP 2). Our frontal polar network was mostly located in FP 1 and may, therefore, be primarily associated with memory-related processes.

The difference between the identified networks in the univariate and multivariate analyses might seem counterintuitive at first but can be explained by the differences in objective and methodology of both analyses. This discrepancy is in line with the observation that significant group-level differences do not necessarily translate to high classification accuracies because of strongly overlapping distributions and different goals of the analysis ${ }^{18,19}$. A significant $P$-value in a group-level analysis does not have to correspond to the ability of distinguishing between individual patients because the statistically significant difference in average values might show low effect sizes. In these case classification performance will be low. In addition, the goal of statistical inference is the identification of localized differences between groups while the goal of classification is to find the best multivariate combination of data, which would allow to generalize the effect to new subjects. These are two inherently different goals which therefore can lead to different outcomes.

In contrast to our results, previous studies that have used univariate analysis of structural MRI and task-based fMRI data have primarily pointed to pre-treatment differences in the anterior cingulate cortex, amygdala, hippocampus, and insula ${ }^{13-17,32-35}$. However, direct comparison with our study is difficult since there are numerous differences between our study and those previous studies. For example, most studies that investigated structural MRI data used a predefined region of interest approach $^{26,32,33,35}$ instead of a whole-brain approach. Most fMRI studies have investigated task-induced changes instead of investigating resting-state fMRI recordings ${ }^{13-17}$. And finally, different types of psychotherapies (such as prolonged exposure therapy), different PTSD 


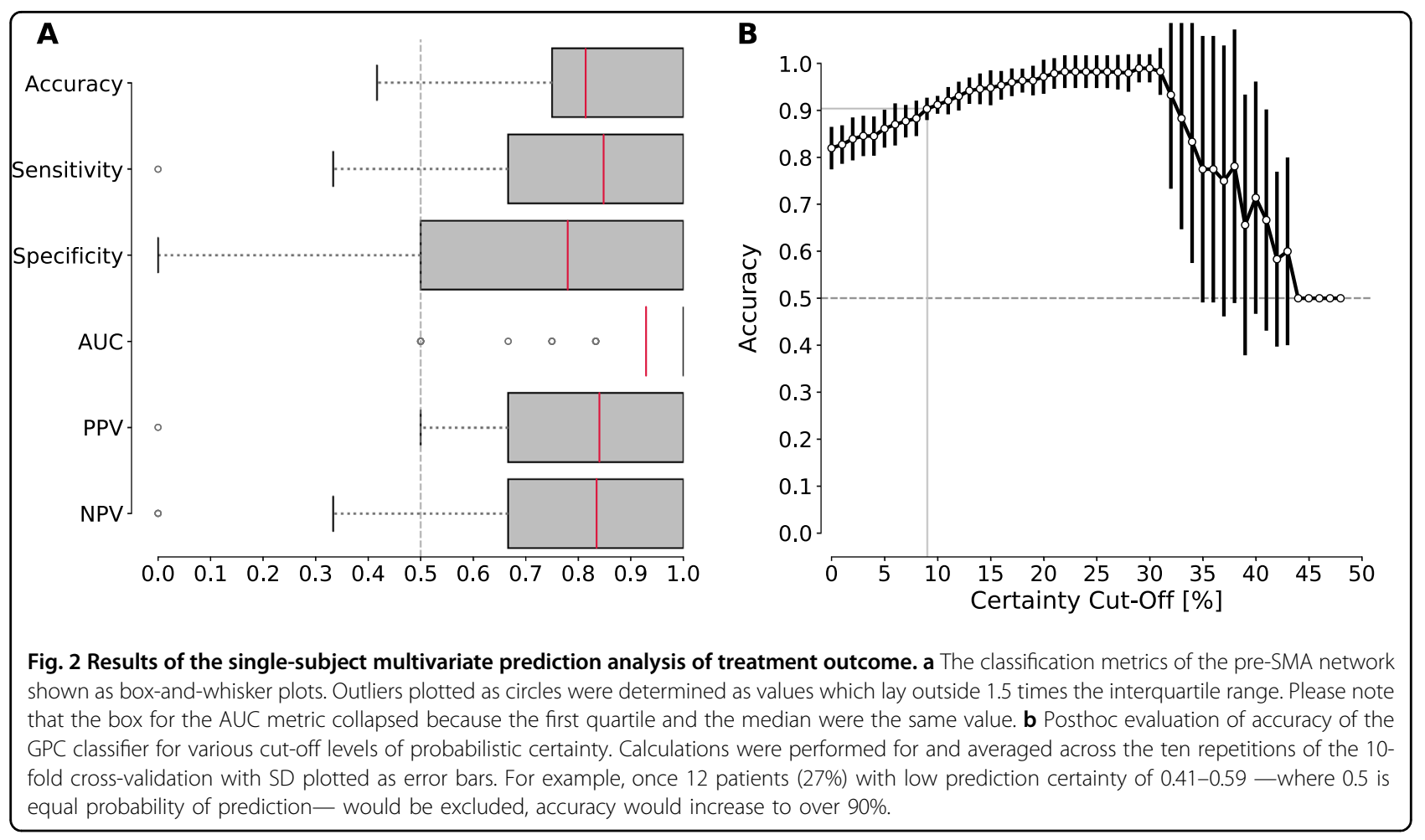

Table 2 Most frequently selected features during the nested-cross-validation procedure of the pre-SMA network.

\begin{tabular}{|c|c|c|c|}
\hline Number of voxels & Max frequency within cluster (\%) & MNI coordinates of max value (mm) & Region name \\
\hline 14 & 99 & $-52,8,-34$ & Left inferior temporal gyrus \\
\hline 10 & 100 & $-24,60,22$ & Left superior frontal gyrus \\
\hline 9 & 100 & $64,4,14$ & Right precentral gyrus \\
\hline 7 & 100 & $-44,8,-14$ & Left insula, left superior temporal pole \\
\hline 6 & 93 & $28,-80,50$ & Right superior parietal lobule \\
\hline 6 & 100 & $0,-4,-2$ & Hypothalamus \\
\hline 4 & 98 & $0,36,58$ & Left medial frontal gyrus \\
\hline 4 & 89 & $32,64,6$ & Right middle frontal gyrus \\
\hline 4 & 96 & $48,-76,18$ & Right middle occipital gyrus \\
\hline 2 & 92 & $0,-80,46$ & Left precuneus \\
\hline 2 & 76 & $40,-84,26$ & Right middle occipital gyrus \\
\hline 2 & 67 & $-44,56,2$ & Left middle frontal gyrus \\
\hline 2 & 75 & $48,52,-6$ & Right middle orbitofrontal gyrus \\
\hline 2 & 63 & $36,44,-18$ & Right inferior orbitofrontal gyrus \\
\hline 1 & 84 & $40,56,-6$ & Right middle orbitofrontal gyrus \\
\hline 1 & 100 & $32,64,14$ & Right superior frontal gyrus \\
\hline 1 & 67 & $-4,68,-10$ & Left medial orbitofrontal gyrus \\
\hline 1 & 57 & $4,-88,34$ & Left cuneus \\
\hline 1 & 69 & $28,8,66$ & Right superior frontal gyrus \\
\hline
\end{tabular}




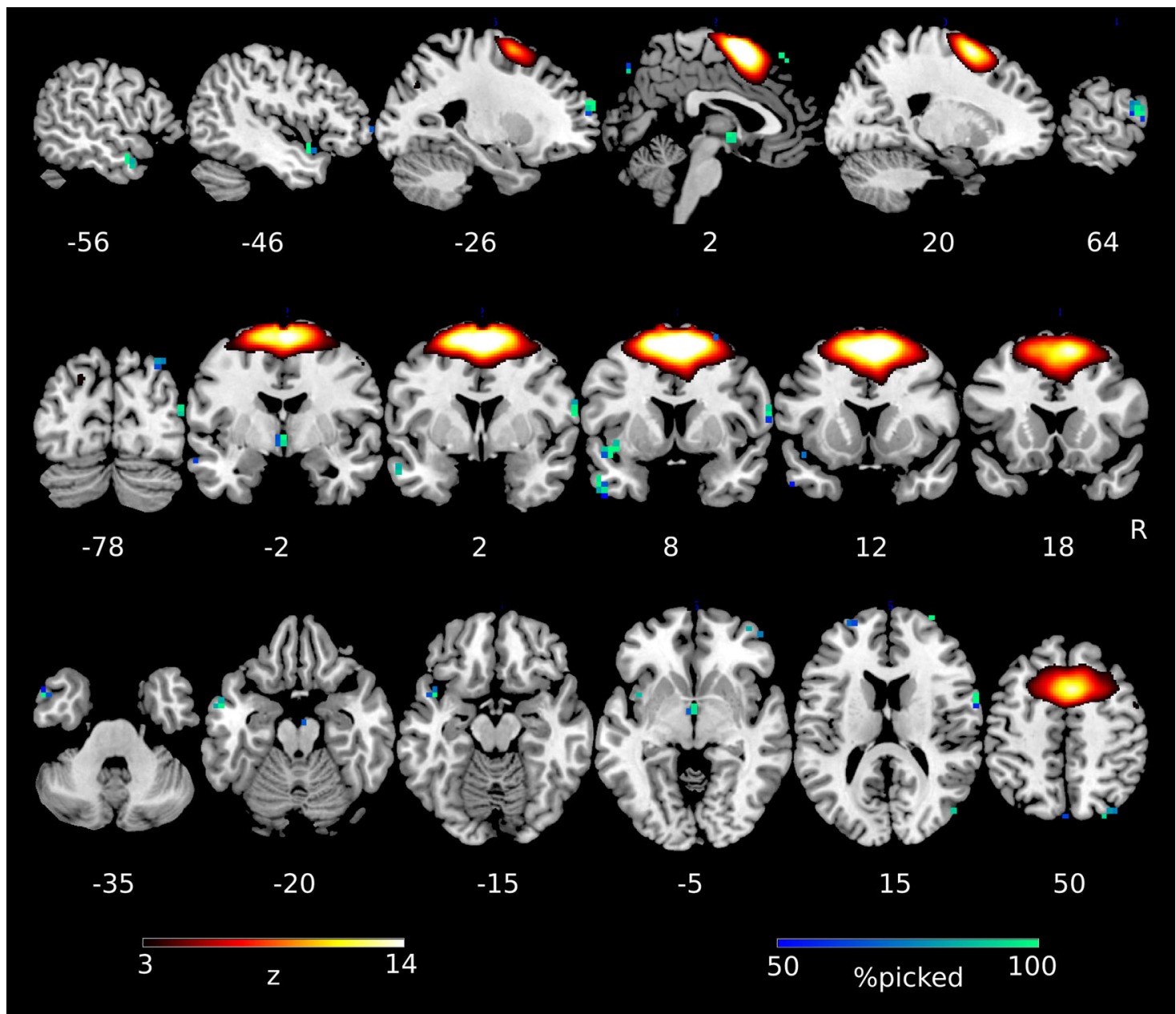

Fig. 3 Best performing network in the multivariate classification (pre-SMA) in hot colors and the most often selected voxels during the classification in cold colors.

populations and experienced trauma, and different treatment-criteria make a direct comparison challenging ${ }^{55}$.

This can be exemplified with the absence of results for the structural MRI analysis which is in contrast to our previous finding of differences in hippocampal volume between patients with remitted vs. persistent $\mathrm{PTSD}^{26}$. This difference could be due to the calculation of the volumes: in the present study, a VBM analysis was employed to provide a highly multivariate data set that could be optimally used during the classification procedure, whereas we previously estimated hippocampal volume using segmentation in Freesurfer. In addition, in this study we chose to focus on treatment response while previously we investigated the more stringent criterion of treatment remission to focus on PTSD persistence. Finally, the previous study was employing a repeatedmeasures design combining pre- and post-treatment data while the current study only focused on the pretreatment data.
The current study has several limitations. The sample size in the current study is small for a machine learning application. This could result in high variance of the estimated accuracy and the results, therefore, require further validation and replication in independent samples $^{56}$. Another limitation of this study is the use of an allmale veteran sample. This limits the generalization of the results to other patients with PTSD. Therefore, a replication of the proposed approach in a more diverse sample would be desirable. Finally, the treatments received by the patients represent a heterogeneous mix of different trauma-focused psychotherapies. While they are considered as first-line treatments and the fact that in realistic settings multiple treatments might be employed by therapists, the results are not specific to one particular treatment. Therefore, the current approach might obscure specific individual patient-by-treatment interactions. Future studies should aim to determine the most optimal treatment for each patient. 
In conclusion, the current study shows that treatment response to trauma-focused psychotherapy can be predicted for individual patients with PTSD using machine learning analysis of rs-fMRI data. This proof-of-concept study demonstrates the feasibility to develop neuroimaging biomarkers for treatment response, which will enhance the personalized treatment of patients with PTSD.

\section{Acknowledgements}

This study was supported by the Dutch Ministry of Defence, the Netherlands Organization for Scientific Research (NWO/ZonMW Vidi 016.156.318) and the AMC Research Council (150622).

\section{Author details}

'Department of Psychiatry, Amsterdam UMC, Location AMC, University of Amsterdam, Amsterdam Neuroscience, Amsterdam, The Netherlands.

${ }^{2}$ Amsterdam Brain and Cognition, University of Amsterdam, Amsterdam, The Netherlands. ${ }^{3}$ ARQ National Psychotrauma Centre, Diemen, The Netherlands. ${ }^{4}$ Department of Psychiatry and Behavioral Sciences, Emory University School of Medicine, Atlanta, GA, USA. ${ }^{5}$ Clinical Psychology Department, Utrecht University, Utrecht, The Netherlands. ${ }^{6}$ Utrecht University Medical Center, Rudolf Magnus Institute of Neuroscience, Utrecht, The Netherlands. ${ }^{7}$ Brain Research and Innovation Centre, Ministry of Defence, Utrecht, The Netherlands

\section{Conflict of interest}

The authors declare that they have no conflict of interest.

\section{Publisher's note}

Springer Nature remains neutral with regard to jurisdictional claims in published maps and institutional affiliations.

Supplementary Information accompanies this paper at (https://doi.org/ 10.1038/s41398-019-0663-7)

Received: 12 September 2019 Revised: 30 September 2019 Accepted: 1 November 2019

Published online: 02 December 2019

\section{References}

1. American Psychiatric A. Diagnostic and statistical manual of mental disorders: DSM-IV. (American Psychiatric Association, Washington, DC, 1994).

2. Atwoli, L., Stein, D. J., Koenen, K. C. \& McLaughlin, K. A. Epidemiology of posttraumatic stress disorder: prevalence, correlates and consequences. Curr. Opin. Psychiatry 28, 307-311 (2015).

3. Kessler, R. C. et al. Trauma and PTSD in the WHO World Mental Health Surveys. Eur. J. Psychotraumatol 8(sup5), 1353383 (2017).

4. Richardson, L. K., Frueh, B. C. \& Acierno, R. Prevalence estimates of combatrelated post-traumatic stress disorder: critical review. Aust. N. Z. J. Psychiatry 44, 4-19 (2010).

5. Sundin, J., Fear, N. T., Iversen, A., Rona, R. J. \& Wessely, S. PTSD after deployment to Iraq: conflicting rates, conflicting claims. Psychol. Med. 40, 367-382 (2010).

6. Cusack, K. et al. Psychological treatments for adults with posttraumatic stress disorder: A systematic review and meta-analysis. Clin. Psychol. Rev. 43, 128-141 (2016).

7. Schnyder, U. et al. Psychotherapies for PTSD: what do they have in common? Eur. J. Psychotraumatol 6, 28186 (2015).

8. Bradley, R., Greene, J., Russ, E., Dutra, L. \& Westen, D. A multidimensional metaanalysis of psychotherapy for PTSD. Am. J. Psychiatry 162, 214-227 (2005).

9. Koch, S. B. et al. Aberrant resting-state brain activity in posttraumatic stress disorder: a meta-analysis and systematic review. Depress Anxiety 33, 592-605 (2016).

10. Fenster, R. J., Lebois, L. A. M., Ressler, K. J. \& Suh, J. Brain circuit dysfunction in post-traumatic stress disorder: from mouse to man. Nat. Rev. Neurosci. 19, 535-551 (2018).

11. Shin, L. M. \& Liberzon, I. The neurocircuitry of fear, stress, and anxiety disorders. Neuropsychopharmacology 35, 169-191 (2010).
12. Rauch, S. L., Shin, L. M. \& Phelps, E. A. Neurocircuitry models of posttraumatic stress disorder and extinction: human neuroimaging research-past, present, and future. Biol. Psychiatry 60, 376-382 (2006).

13. van Rooij, S. J., Geuze, E., Kennis, M., Rademaker, A. R. \& Vink, M. Neura correlates of inhibition and contextual cue processing related to treatment response in PTSD. Neuropsychopharmacology 40, 667-675 (2015).

14. Aupperle, R. L. et al. Neural responses during emotional processing before and after cognitive trauma therapy for battered women. Psychiatry Res. 214, 48-55 (2013).

15. Falconer, E., Allen, A., Felmingham, K. L., Williams, L. M. \& Bryant, R. A. Inhibitory neural activity predicts response to cognitive-behavioral therapy for posttraumatic stress disorder. J. Clin. Psychiatry 74, 895-901 (2013).

16. Fonzo, G. A. et al. PTSD Psychotherapy Outcome Predicted by Brain Activation During Emotional Reactivity and Regulation. Am. J. Psychiatry 174, 1163-1174 (2017).

17. Bryant, R. A. et al. Amygdala and ventral anterior cingulate activation predicts treatment response to cognitive behaviour therapy for post-traumatic stress disorder. Psychol. Med. 38, 555-561 (2008).

18. Bzdok, D., loannidis, J. P. A. Exploration, inference, and prediction in neuroscience and biomedicine. Trends Neurosci. 42, 251-262 (2019).

19. Arbabshirani, M. R., Plis, S., Sui, J. \& Calhoun, V. D. Single subject prediction of brain disorders in neuroimaging: Promises and pitfalls. Neuroimage 145, 137-165 (2017)

20. Gong, Q. et al. Using structural neuroanatomy to identify trauma survivors with and without post-traumatic stress disorder at the individual level. Psychol. Med. 44, 195-203 (2014).

21. Zhang, Q. et al. Multimodal MRI-based classification of trauma survivors with and without post-traumatic stress disorder. Front Neurosci. 10, 292 (2016).

22. Li, X. et al. Dynamic functional connectomics signatures for characterization and differentiation of PTSD patients. Hum. Brain Mapp. 35, 1761-1778 (2014).

23. Liu, F. et al. Characterization of post-traumatic stress disorder using restingstate fMRI with a multi-level parametric classification approach. Brain Topogr. 28, 221-237 (2015).

24. Yuan, M. et al. Pre-treatment resting-state functional MR imaging predicts the long-term clinical outcome after short-term paroxtine treatment in posttraumatic stress disorder. Front Psychiatry 9, 532 (2018).

25. Etkin A., et al. Using fMRI connectivity to define a treatment-resistant form of post-traumatic stress disorder. Sci. Transl. Med. 11, pii: eaal3236 (2019).

26. van Rooij, S. J. et al. Smaller hippocampal volume as a vulnerability factor for the persistence of post-traumatic stress disorder. Psychol. Med. 45, 2737-2746 (2015).

27. Kennis, M. et al. Treatment outcome-related white matter differences in veterans with posttraumatic stress disorder. Neuropsychopharmacology $\mathbf{4 0}$, 2434-2442 (2015)

28. van Rooij, S. J., Kennis, M., Vink, M. \& Geuze, E. Predicting treatment outcome in PTSD: a longitudinal functional MRI Study on trauma-unrelated emotional processing. Neuropsychopharmacology 41, 1156-1165 (2016).

29. Kennis, M., Rademaker, A. R., van Rooij, S. J., Kahn, R. S. \& Geuze, E. Resting state functional connectivity of the anterior cingulate cortex in veterans with and without post-traumatic stress disorder. Hum. Brain Mapp. 36, 99-109 (2015).

30. Kennis, M., van Rooij, S. J., van den Heuvel, M. P., Kahn, R. S. \& Geuze, E. Functional network topology associated with posttraumatic stress disorder in veterans. Neuroimage Clin. 10, 302-309 (2016).

31. Nickerson, L. D., Smith, S. M., Ongur, D. \& Beckmann, C. F. Using dual regression to investigate network shape and amplitude in functional connectivity analyses. Front Neurosci. 11, 115 (2017).

32. Bryant, R. A. et al. Rostral anterior cingulate volume predicts treatment response to cognitive-behavioural therapy for posttraumatic stress disorder. J. Psychiatry Neurosci. 33, 142-146 (2008).

33. Helpman, L. et al. Ptsd remission after Prolonged exposure treatment is associated with anterior cingulate cortex thinning and volume reduction Depress Anxiety 33, 384-391 (2016).

34. Nardo, D. et al. Gray matter density in limbic and paralimbic cortices is associated with trauma load and EMDR outcome in PTSD patients. J. Psychiatr. Res. 44, 477-485 (2010)

35. Rubin, M. et al. Greater hippocampal volume is associated with PTSD treatment response. Psychiatry Res. Neuroimaging 252, 36-39 (2016).

36. Blake, D. D. et al. The development of a Clinician-Administered PTSD Scale. J. Trauma Stress 8, 75-90 (1995).

37. First M. B., Spitzer R. L., Gibbon M. \& Williams J. B. Structured clinical interview for DSM-IV axis I disorders. (New York State Psychiatric Institute, New York 1995). 
38. Brady, K. et al. Efficacy and safety of sertraline treatment of posttraumatic stress disorder: a randomized controlled trial. JAMA 283, 1837-1844 (2000).

39. Davidson, J. R., Rothbaum, B. O., van der Kolk, B. A., Sikes, C. R. \& Farfel, G. M. Multicenter, double-blind comparison of sertraline and placebo in the treatment of posttraumatic stress disorder. Arch. Gen. Psychiatry 58, 485-492 (2001).

40. World Medical A. World Medical Association Declaration of Helsinki: ethical principles for medical research involving human subjects. JAMA $\mathbf{3 1 0}$, 2191-2194 (2013).

41. Pruim, R. H. R. et al. ICA-AROMA: a robust ICA-based strategy for removing motion artifacts from fMRI data. Neuroimage 112, 267-277 (2015).

42. Beckmann, C. F. \& Smith, S. M. Probabilistic independent component analysis for functional magnetic resonance imaging. IEEE Trans. Med. Imaging 23, 137-152 (2004)

43. Abou Elseoud, A. et al. Group-ICA model order highlights patterns of functional brain connectivity. Front Syst. Neurosci. 5, 37 (2011).

44. Biswal, B. B. et al. Toward discovery science of human brain function. Proc. Natl Acad. Sci. USA 107, 4734-4739 (2010).

45. Smith, S. M. \& Nichols, T. E. Threshold-free cluster enhancement: addressing problems of smoothing, threshold dependence and localisation in cluster inference. Neuroimage 44, 83-98 (2009).

46. Rasmussen C. E., Williams C. K. I. Gaussian Processes for Machine Learning. (The MIT Press, Cambridge, Massachusetts, 2006).
47. Marquand, A. et al. Quantitative prediction of subjective pain intensity from whole-brain fMRI data using Gaussian processes. Neuroimage 49, 2178-2189 (2010).

48. Haufe, S. et al. On the interpretation of weight vectors of linear models in multivariate neuroimaging. Neuroimage 87, 96-110 (2014).

49. Ruan, J. et al. Cytoarchitecture, probability maps, and functions of the human supplementary and pre-supplementary motor areas. Brain Struct. Funct. 223, 4169-4186 (2018).

50. Eickhoff, S. B. et al. Co-activation patterns distinguish cortical modules, their connectivity and functional differentiation. Neuroimage 57, 938-949 (2011).

51. van Rooij, S. J. H. \& Jovanovic, T. Impaired inhibition as an intermediate phenotype for PTSD risk and treatment response. Prog. Neuropsychopharmacol. Biol. Psychiatry 89, 435-445 (2019).

52. van Waarde, J. A. et al. A functional MRI marker may predict the outcome of electroconvulsive therapy in severe and treatment-resistant depression. Mol. Psychiatry 20, 609-614 (2015).

53. Bludau, S. et al. Cytoarchitecture, probability maps and functions of the human frontal pole. Neuroimage 93(Pt 2), 260-275 (2014).

54. Gilbert, S. J. et al. Functional specialization within rostral prefrontal cortex (area 10): a meta-analysis. J. Cogn. Neurosci. 18, 932-948 (2006).

55. Colvonen, P. J. et al. Pretreatment biomarkers predicting PTSD psychotherapy outcomes: a systematic review. Neurosci. Biobehav Rev. 75, 140-156 (2017).

56. Varoquaux, G. Cross-validation failure: Small sample sizes lead to large error bars. Neuroimage 180(Pt A), 68-77 (2018). 